\title{
Computational Study of Photoexcited Dynamics in Bichromophoric Cross-Shaped Oligofluorene
}

\author{
D. Ondarse-Alvarez, ${ }^{\dagger}$ N. Oldani, ${ }^{\dagger}$ S. Tretiak, ${ }^{\text {,I }}$ and S. Fernandez-Alberti ${ }^{*}{ }^{\dagger}$ \\ ${ }^{\dagger}$ Universidad Nacional de Quilmes, Roque Saenz Peña 352, B1876BXD Bernal, Argentina \\ ${ }^{I}$ Theoretical Division, Center for Nonlinear Studies (CNLS), and Center for Integrated Nanotechnologies (CINT), Los Alamos \\ National Laboratory, Los Alamos, New Mexico 87545, United States
}

\begin{abstract}
The non-adiabatic excited state molecular dynamics (NA-ESMD) approach is applied to investigate photoexcited dynamics and relaxation pathways in a spiro-linked conjugated polyfluorene at room $(T=300 \mathrm{~K})$ and low $(T=10 \mathrm{~K})$ temperatures. This dimeric aggregate consists of two perpendicularly oriented weakly interacting $\alpha$-polyfluorene oligomers. The negligible coupling between the monomer chains results in an initial absorption band composed of equal contributions of the two lowest excited electronic states, each localized on one of the two chains. After photoexcitation, an efficient ultrafast localization of the entire electronic population to the lowest excited state is observed on the time scale of about $100 \mathrm{fs}$. Both internal conversion between excited electronic states and vibronic energy relaxation on a single electronic state contribute to this process. Thus, photoexcited dynamics of the polyfluorene dimer follows two distinct pathways with substantial temperature dependence on their efficiency. One relaxation channel involves resonance electronic energy transfer between the monomer chains, whereas the second pathway concerns the relaxation of the electronic energy on the same chain that has been initially excited due to electron-phonon coupling. Despite the slower vibrational relaxation, a more efficient ultrafast electronic relaxation is observed at low temperature. Our numerical simulations analyze the effects of molecular geometry distortion during the electronic energy redistribution and suggest spectroscopic signatures reflecting complex electron-vibrational dynamics.
\end{abstract}

\section{INTRODUCTION}

Light-emitting polymers (LEPs) have attracted considerable interest worldwide due to their practical applications in a large variety of optoelectronic devices and their multiple potential advantages concerning preparation and operation over other display and lighting technologies. ${ }^{1-8}$ A great variety of LEPs have been developed, and their physicochemical properties have been well characterized. Among them, we can mention LEPs based on poly( $p$-phenylenevinylene) (PPV), poly $(p$ phenylene) (PPP), polyacetylene, polyaniline, polypyrrole, polyfluorene, and polythiophene structural motifs.

The color and quantum efficiency of light emission, turn-on voltage, and oxidation and thermal stability of the LEP devices must be optimized to be applicable as commercial lightemitting devices. In the last decades, considerable progress has been made with chemical modification and copolymerization of conjugated polymers to tune light emission from the near-UV to near-red light range. ${ }^{9-11}$ Emission over the entire visible spectrum has been achieved with impressive efficiency and brightness. $^{12-15}$ Within these developments, blue-emitting polymers and oligomers have received special attention due to their potential applications as color converters and full color displays that complete the luminescent spectrum. Blue emission of LEPs needs large band gaps in the polymers. Conjugated polyfluorenes (PFs) are structurally suitable for this purpose. ${ }^{16-21}$ The rigid planarized biphenyl structure bridged by a methylene group in the fluorene monomer unit furnishes them a wide band gap ensuring blue emission. In addition, its C-9 position provides a facile functionalization for tuning the solubility, optical, and electrochemical properties, processability of polymers, and intermolecular interactions. Fluorene-based conjugated oligomers can emit blue light at an efficiency of up to $99 \%$ in solution and $90 \%$ in solid films.

A considerable amount of theoretical and experimental studies have been focused on the design of LEDs involving PFs as the active layer. ${ }^{22-25}$ PF-based devices present attractive current-voltage-luminance characteristics that have triggered development of several procedures for their manufacture, as well as fundamental studies of their electronic properties. Different strategies have been set up to improve the performance of the devices. Among them, we can mention the addition of side groups as simple acceptor and/or donor groups like diamino or dicyano substituents, ${ }^{26}$ extension of the conjugation length using indenofluorene units, ${ }^{22}$ improvement of their water solubility, ${ }^{27}$ end-capping, ${ }^{28}$ enforcement of the planar structure through ladder-type products, ${ }^{21}$ and the incorporation of a large variety of periodic table elements like rare earth and metal complexes. ${ }^{29,30}$ That is, substituted PFs have been developed in order to enhance specific properties

Received: July 22, 2014

Revised: September 6, 2014

Published: October 23, 2014 
deemed attractive for a particular application or designed for testing fundamental concepts.

Nowadays, blue-emitting materials composed of conjugated polyfluorenes (PFs) can be designed and synthesized for electronic applications like organic light-emitting diodes (OLEDs), field-effect transistors, and solar cells. ${ }^{27,29,31}$ Among the PFs main optical and electrical properties of interest, we can mention their high photoluminescence quantum yields, high thermal stability, relatively good charge transport properties, and tunable light emission throughout the entire visible region. ${ }^{30}$ Besides, PF homopolymers present thermotropic liquid crystallinity, which allows their ordered organization on layers. ${ }^{32,33}$

However, PF-based light-emitting diodes (LEDs) can also exhibit low-energy emission bands that convert the desired blue emission to green or yellow. This red-shifted and thus less efficient emission has been a key drawback for PFs with planar $\pi$-system, causing problems of color purity and stability of the light emitted by these LEDs. The long wavelength tail in the emission spectra may be caused by device degradation under fabrication or operation, generating fluorenone defects by photo-oxidization, or thermal oxidation. ${ }^{34,35}$ On the other hand, the poor spectral stability of PFs may be attributed to molecular aggregation and/or excimer formation. ${ }^{18,35-41}$ The suppression of the excimer emission can be achieved by introducing lowband gap chromophore groups, like anthracenes, and perylenes, into the backbone of PFs. ${ }^{42-45}$ Alternatively, aggregation can be prevented by attaching a variety of side chain structures like polyphenylene, ${ }^{46}$ Fréchet-type dendron substituents, ${ }^{47}$ or Müllen-type dendrimers. ${ }^{48}$ The use of bulky dendrimer substituents permits spatial control of the PF chain and hinders aggregation. Additionally, these substituents serve as solubilizing groups. These are a few synthetic approaches developed to enhance thermal stability of polymers in amorphous films as well as to improve desired emission properties.

A different strategy to suppress the formation of interchain aggregates consists of introducing spirofluorene structures into PFs. ${ }^{46,49-55}$ Spiro-linked PFs utilize a spiro-bridge to connect two conjugated moieties. The tetrahedral character of the $\mathrm{sp}^{3}$ hybridized carbon atom that connects the moieties maintains the perpendicular arrangement of the two molecular halves. This structural feature efficiently reduces the dense $\pi-\pi$ interchain stacking in the films and increases the PFs solubility. Besides, the resulting unwieldy structures also contribute to suppression of the excimer formation. Moreover, the higher molecular weight and steric demand of the rigid cross-shaped molecular structure led to the frequently desired increase of the glass transition temperature compared with the corresponding monomer. $^{49}$

In this article, using numerical simulations we analyze the electron-vibrational energy relaxation that takes place after the photoexcitation of a spiro-linked homodimeric PF. ${ }^{56}$ The interchromophore and intrachromophore relaxation pathways are identified that arise from a weak interaction between the monomer chains and well as vibronic couplings. Our numerical modeling evaluates the respective relaxation time scales at room $(T=300 \mathrm{~K})$ and low $(T=10 \mathrm{~K})$ temperatures leading to a localization of the excited state wave function on a segment of the single oligomer forming the final fluorescent state which is responsible for a light emission. Effect of coupling to molecular vibrations and conformations as well as spectroscopic signatures of observed dynamical processes are also identified. Our computations use the recently developed non-adiabatic excited state molecular dynamics (NA-ESMD) method. ${ }^{57-60}$ This approach has been previously successfully applied to a variety of conjugated molecular systems providing a detailed picture of internal conversion processes following photoexcitation. $^{61-63,58}$

The paper is organized as follows. Section II provides a brief overview of the numerical NA-ESMD approach, electronic transition densities calculation, and computational details. In Section III we present and discuss our results. Finally, Section IV summarizes our findings and conclusions.

\section{METHODS}

A. NA-ESMD Background. The NA-ESMD code $57,64,65$ allows for a direct modeling of nonadiabatic molecular dynamics ${ }^{66}$ that has been specifically developed to simulate the photoexcitation and subsequent electronic and vibrational relaxation processes in extended conjugated molecules involving multiple coupled electronic excited states. The NAESMD makes use of the MDQT (Molecular Dynamics with Quantum Transitions) algorithm developed by Tully ${ }^{67-69}$ augmented with a numerical procedure able to deal with trivial unavoided crossings between noninteracting adiabatic states ${ }^{70}$ and empirical corrections accounting for electronic decoherences. ${ }^{71}$ The nuclei degrees of freedom are treated classically using the Langevin equation at constant temperature, while the electronic wave function $\Psi(\mathbf{r}, \mathbf{R}, t)$ is propagated quantum mechanically in the discrete basis representation of the adiabatic electronic states $\phi_{\alpha}(\mathbf{r} ; \mathbf{R}(t))$ of the molecule as

$$
\psi(\mathbf{r}, \mathbf{R}, t)=\sum_{\alpha} c_{\alpha}(t) \phi_{\alpha}(\mathbf{r} ; \mathbf{R}(t))
$$

with $c_{\alpha}(t)$ being the time-dependent expansion coefficients, and $\mathbf{r}$ and $\mathbf{R}$ the electronic and nuclear coordinates, respectively. Therefore, the coupled equations of motion for the $c_{\alpha}(t)$ coefficients are obtained by substituting eq 1 into the timedependent Schrödinger equation yielding the following expression

$$
i \hbar \frac{\partial c_{\alpha}(t)}{\partial t}=c_{\alpha}(t) E_{\alpha}-i \hbar \sum_{\beta \neq \alpha} c_{\beta}(t) \dot{\mathbf{R}} \cdot \mathbf{d}_{\alpha \beta}
$$

where $E_{\alpha}$ is the energy of the $\alpha$ th adiabatic state calculated at time $t, \mathbf{d}_{\alpha \beta}$ is the nonadiabatic coupling vector(NACR) defined by $\mathbf{d}_{\alpha \beta}=\left\langle\phi_{\alpha}(\mathbf{r} ; \mathbf{R}(t)) \mid \nabla_{\mathbf{R}} \phi_{\beta}(\mathbf{r} ; \mathbf{R}(t))\right\rangle$, and

$$
\dot{\mathbf{R}} \cdot \mathbf{d}_{\alpha \beta}=\left\langle\phi_{\alpha} \mid \frac{\partial \phi_{\beta}}{\partial t}\right\rangle
$$

is the time-dependent nonadiabatic coupling (NACT).

Excited state energies, ${ }^{72-74}$ gradients, ${ }^{57,75,76}$ and nonadiabatic coupling ${ }^{57,77-79}$ terms are calculated on the fly using the collective electron oscillator (CEO) approach. ${ }^{80-83}$ For all simulations presented here, we use the Austin model 1 $(\mathrm{AM} 1)^{84}$ semiempirical level in combination with the configuration interaction singles (CIS) formalism to describe correlated excited states. A detailed discussion of the NAESMD implementation, computational procedure, advantages, and testing parameters can be found elsewhere. ${ }^{57-60}$

B. Analysis of Transient Electronic Transition Density. Within the CEO approach, ${ }^{85,86}$ changes in the distribution of the electronic density induced by photoexcitation from the ground state $g$ to the current excited electronic state $\alpha$ are followed through the diagonal elements of the transition 


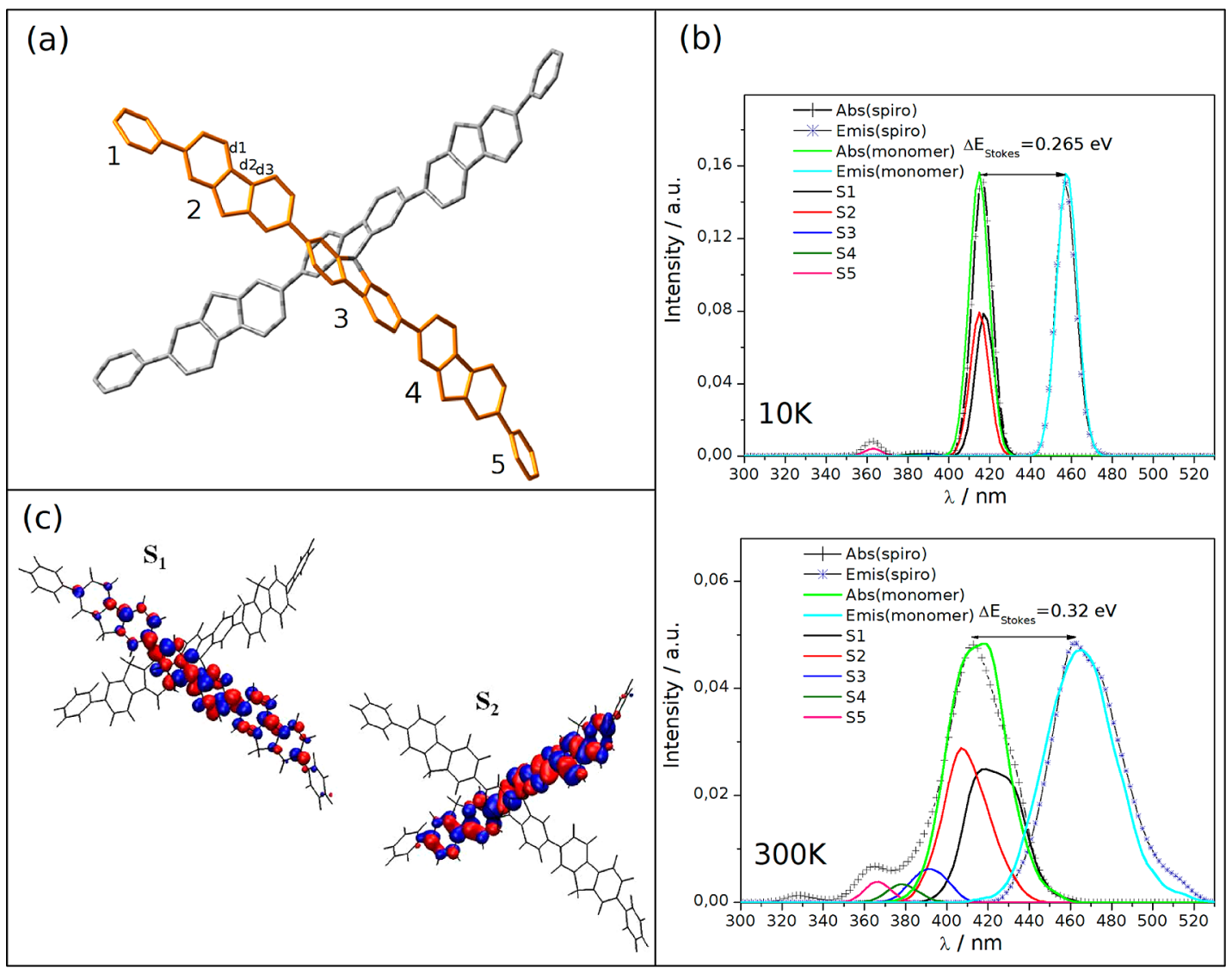

Figure 1. (a) Chemical structure of the spiro-linked polyfluorene. (b) Simulated absorption and emission spectra at 10 and $300 \mathrm{~K}$ with separated contributions of different excited states to the absorption spectrum. (c) Initial localization of the electronic transition densities for the two lowest excited states.

density matrices ${ }^{87} \rho_{\mathrm{nm}}^{g \alpha}(t) \equiv\left\langle\phi_{\alpha}(\mathbf{r} ; \mathbf{R}(t))\left|c_{m}^{+} c_{n}\right| \phi_{g}(\mathbf{r} ; \mathbf{R}(t))\right\rangle$ (denoted electronic normal modes). Here $c_{m}^{+}\left(c_{n}\right)$ represent the creation (annihilation) operators; and $n$ and $m$ refer to atomic orbital (AO) basis functions. In accordance with the required normalization condition $\left(\rho^{g \alpha}(t)\right)^{2}=\sum_{\mathrm{n}, \mathrm{m}}\left(\rho_{\mathrm{nm}}^{g \alpha}(t)\right)^{2}=1$ fulfilled within the CIS approximation ${ }^{74}$ and the fact that the final transition density is well localized on one of the monomers, the transition density localized on each of the monomers at each time of the NA-ESMD simulations can be written as

$$
\left(\rho_{\mathrm{E} / \mathrm{NE}}^{g \alpha}(t)\right)^{2}=\sum_{\mathrm{n}_{\mathrm{A}} \mathrm{m}_{\mathrm{A}}}\left(\rho_{\mathrm{n}_{\mathrm{A}} \mathrm{m}_{\mathrm{A}}}^{g \alpha}(t)\right)^{2}+\frac{1}{2} \sum_{\mathrm{n}_{\mathrm{B}} \mathrm{m}_{\mathrm{B}} \in \mathrm{C}_{\text {bridge }}}\left(\rho_{\mathrm{n}_{\mathrm{B}} \mathrm{m}_{\mathrm{B}}}^{g \alpha}(t)\right)^{2}
$$

where the subindex $\mathrm{E} / \mathrm{NE}$ indicates the monomer that emit $(\mathrm{E})$ or do not emit (NE) at long times of the NA-ESMD trajectory. The index A runs over all atoms localized in the corresponding monomer $\mathrm{E}$ or $\mathrm{NE}$, and the index $\mathrm{B}$ runs over carbon $\mathrm{C}_{\text {bridge }}$ atom that acts as bridge between them. Using that, we can define the monomer participation number ${ }^{8,89}$ as

$$
\mathbf{P}(t)=\left(\left(\left(\rho_{\mathrm{E}}^{g \alpha}(t)\right)^{2}\right)^{2}+\left(+\left(\rho_{\mathrm{NE}}^{g \alpha}(t)\right)^{2}\right)^{2}\right)^{-1}
$$

Values of $\left(\rho_{\mathrm{E} / \mathrm{NE}}^{g \alpha}(t)\right)^{2} \approx 1$ (i.e., $\left.\left(\rho_{\mathrm{NE} / \mathrm{E}}^{g \alpha}(t)\right)^{2}=0\right)$, indicating a complete localization of the transition density in the $\mathrm{E} / \mathrm{NE}$ monomer, lead to values of $\mathbf{P}(t) \approx 1$, while values of $\left(\rho_{\mathrm{E} / \mathrm{NE}}^{g \alpha}(t)\right)^{2} \approx 0.5$, corresponding to transition densities fully delocalized between both monomers, lead to values of $\mathbf{P}(t) \approx 2$.

C. Molecular Dynamics Simulations. The spiro-linked conjugated polyfluorene dimer studied in this work is depicted in Figure 1. First, 1 ns of ground state molecular dynamics simulations at 10 and $300 \mathrm{~K}$ have been performed using a Langevin friction coefficient $\gamma$ of $2.0 \mathrm{ps}^{-1}$. Snapshots of 400 nuclei positions and momenta (configurational space) have been collected from the last $0.6 \mathrm{~ns}$ and used as initial conditions for the subsequent NA-ESMD simulations. The NA-ESMD trajectories have started from these initial configurations by instantaneously promoting the system to an initial excited state $\alpha$, with the frequency $\Omega_{\alpha}$, selected according to a FranckCondon window defined as $\mathrm{g}_{\alpha}(\mathbf{r}, \mathbf{R})=f_{\alpha} \exp \left[-T^{2}\left(E_{\text {laser }}-\right.\right.$ $\left.\left.\Omega_{\alpha}\right)^{2}\right] \cdot f_{\alpha}$ represents the normalized oscillator strength for the $\alpha$ state, and $E_{\text {laser }}$, expressed in units of $\mathrm{fs}^{-1}$, as well as $\Omega_{\alpha}$, represents the energy of a laser pulse centered at $415 \mathrm{~nm}$ that corresponds to the middle of the absorption spectrum. A Gaussian laser pulse, $f(t)=\exp \left(-t^{2} / 2 T^{2}\right)$, has been considered with $T^{2}=42.5 \mathrm{fs}$ corresponding to a fwhm (full width at half maximum) of $100 \mathrm{fs}$.

Ten electronic states and their corresponding nonadiabatic couplings have been included in the simulations. The NAESMD trajectories of $500 \mathrm{fs}$ duration are propagated at 10 and $300 \mathrm{~K}$ in order to obtain a reasonable statistics. Classical time steps of $0.5 \mathrm{fs}$ and 0.1 fs have been used for nuclei propagation in the ground and excited state dynamics, respectively. Besides, a quantum time step of $0.025 \mathrm{fs}$ has been used to propagate the electronic degrees of freedom during the NA-ESMD simulations. In order to identify and to deal with trivial unavoided crossings, the quantum time step was further reduced by a factor of 40 . More details concerning the NA- 
ESMD implementation and parameters can be found elsewhere. $^{57,58,70}$

\section{RESULTS AND DISCUSSION}

Figure 1a shows the structure of the spiro-linked polyfluorene homodimer studied here. Owing to perpendicular orientation,
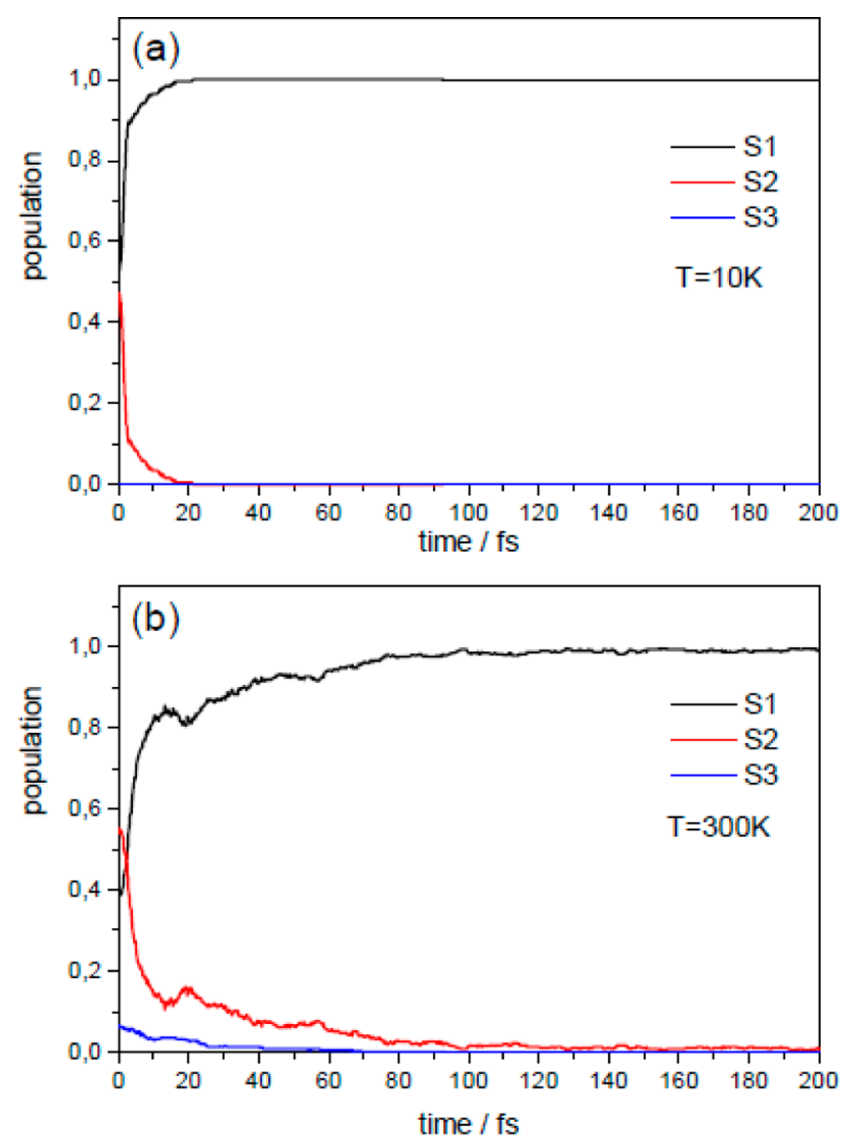

Figure 2. Average population on each electronic surface as a function of time obtained from the fraction of trajectories in each state.

$\alpha$-polyfluorene chains are weakly interacting. The monomers are connected by a common $\mathrm{C}_{\text {bridge }}$ atom in the middle of the central fluorene units, that acts as a bridge between oligomers. The tetrahedral character of $\sigma$-bonds in this $\mathrm{C}_{\text {bridge }}$ atom maintains the relative perpendicular orientation of the monomers.

The negligible coupling between the monomer chains results in an initial absorption band composed of equal contributions of the two lowest electronic excited states. This is shown in Figure $1 \mathrm{~b}$ where the simulated absorption spectra at two different temperatures, 10 and $300 \mathrm{~K}$, are displayed. The analysis of the contributions of the different excited states to the final absorption spectra at $10 \mathrm{~K}$ reveals the quasidegeneracy of the first two excited states. Furthermore, their corresponding absorption bands are very close to the band obtained for the simulation of an individual monomer. Notably, states $S_{1}$ and $S_{2}$ are orthogonal and uncoupled in the dipole approximation. Nevertheless, electrostatic interactions between transition densities on the monomers lead to a small but nonvanishing electronic coupling. In fact, the splitting between transition energies of the two lowest excited states $S_{1}$ and $S_{2}$ in a dimer at its equilibrium ground state geometry allows evaluation of this interaction to be about $5 \mathrm{~cm}^{-1}$. These
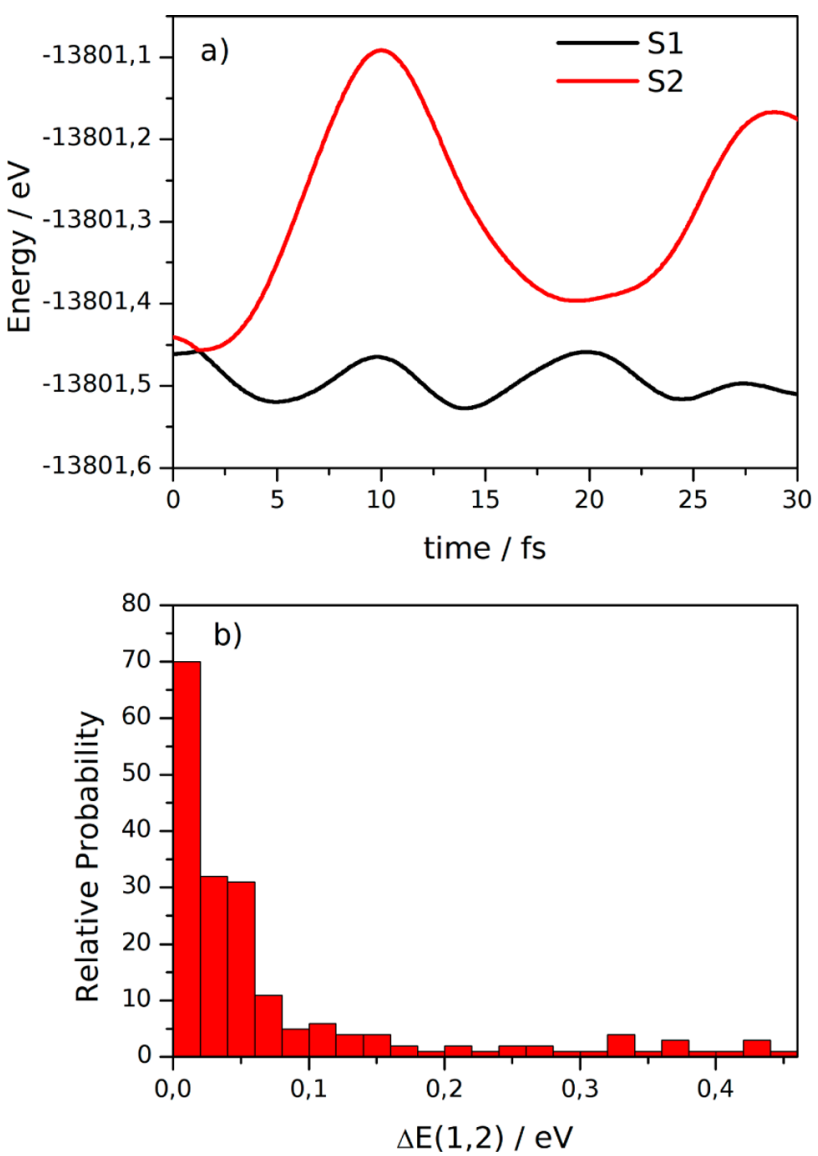

Figure 3. (a) Time-dependence of the energies for the $S_{1}$ and $S_{2}$ states for a typical trajectory at $10 \mathrm{~K}$ is displayed. (b) Distribution of effective $S_{2} \rightarrow S_{1}$ transitions in terms of transition energy $\Delta E$.

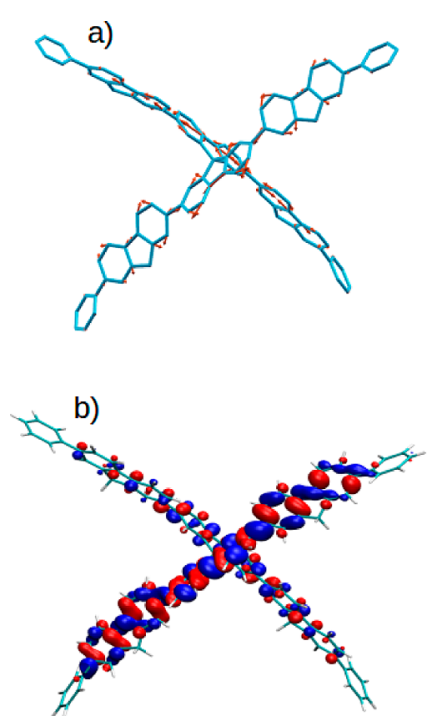

Figure 4. (a) Nonadiabatic coupling vector for a typical trajectory at $10 \mathrm{~K}$ at the time of the effective $S_{2} \rightarrow S_{1}$ transition. (b) Localization of the electronic transition density at the time of the effective $S_{2} \rightarrow S_{1}$ transition.

observations agree with the initial localization of the electronic transition densities for the two lowest excited states as shown in Figure 1c. As can be seen, these states are completely localized on individual molecules. The emission spectra (Figure $1 \mathrm{~b}$ ) are also similar to the spectra corresponding to the individual 


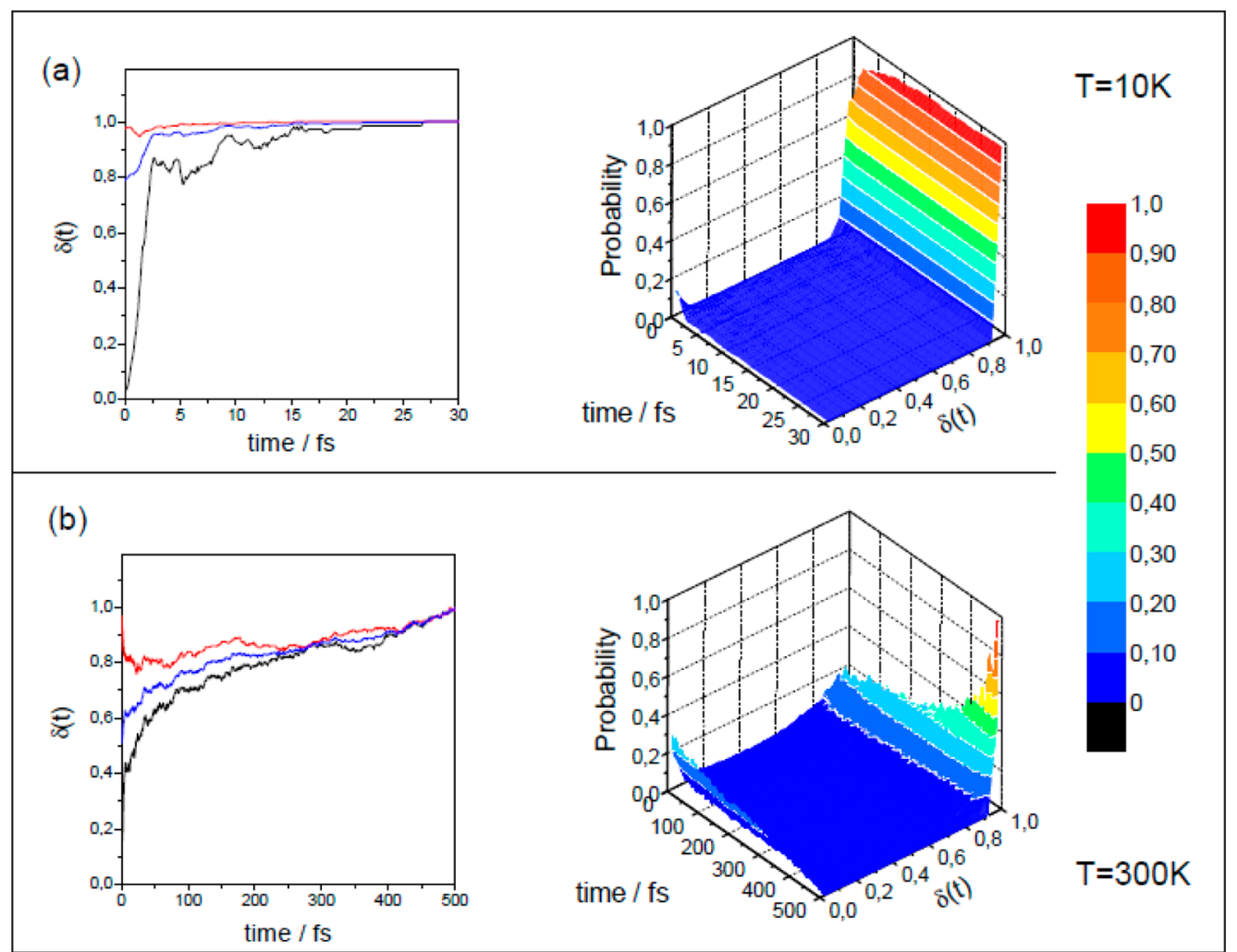

Figure 5. Left: Time evolution of the overlap between the current and final transition densities averaged over the trajectories with the initial transition density localized on the same branch as the final one (red line), averaged over the trajectories with initial transition density localized on the different branch as the final one (black line), and averaged over all the trajectories (blue line). Right: The respective wavepacket dynamics constructed from the evolution over time of the distribution of the overlap between the current and final transition densities: (a) at $10 \mathrm{~K}$ and (b) at $300 \mathrm{~K}$.

monomer, confirming that the spiro-linkage does not affect the UV-visible properties of the original monomers. The observed Stokes shifts (defined as a difference between absorption and emission maxima) of $\sim 0.26$ and $0.32 \mathrm{eV}$, for the spectra at 10 and $300 \mathrm{~K}$, respectively, simply indicate a slower vibrational relaxation at low temperature. These values are expected to approach $0.36 \mathrm{eV}$ corresponding to the fully relaxed excited state equilibrium geometry, ${ }^{56}$ indicating that the relaxation has not been completely achieved after $500 \mathrm{fs}$ of the NA-ESMD simulations due to effect of slow torsional motions. ${ }^{56}$ As expected, the larger conformational space spanned by the two chains at $300 \mathrm{~K}$ results in wider spectra compared to that at low temperature. Overall, these results justify an assumption that the majority of vibrational relaxation takes place over the first picosecond of the dynamics forming the final state contribution to the emission.

To analyze electronic dynamics and vibrational relaxation of the spiro-linked $\mathrm{PF}$, we start with evolution of the average population on $S_{1}$ and $S_{2}$ states as a function of time obtained from the fraction of NA-ESMD trajectories in each state shown in Figure 2. According to the initial excited state selection described in section $\mathrm{C}$, the initial fraction of trajectories has resulted in equal distribution between $S_{1}$ and $S_{2}$ states. With time, an efficient ultrafast migration of the electronic population to the lowest $S_{1}$ state is observed.

After photoexcitation, the molecular system experiences a first ultrafast interchromophore energy transfer. The initially small energy gap between $S_{1}$ and $S_{2}$ decreases until a conical intersection between both states is reached. This can be seen in Figure 3a, where the time-dependence of the energies for the $S_{1}$ and $S_{2}$ states for a typical trajectory at $10 \mathrm{~K}$ is displayed. These trajectories present significant crossing probabilities at earlier times, and they mainly hop from $S_{1}$ to $S_{2}$ in less than 5 fs. Figure $3 b$ shows the distribution of effective $S_{2} \rightarrow S_{1}$ transitions in terms of transition energy. The peak at values less than 0.02 $\mathrm{eV}$ indicates the proximity to the conical intersection seam.

In order to analyze the nuclear motions that contribute to the ultrafast energy transfer, the nonadiabatic coupling vector (NACR) is examined. The direction of NACR can be interpreted as the direction of the force on the nuclei during the transition. ${ }^{90}$ Figure 4 a presents it for a typical trajectory at the time of the effective $S_{2} \rightarrow S_{1}$ transition. These nuclear displacements are related to the nature of the two electronic states involved in the dynamics. Therefore, this interchromophore vibrational energy redistribution is concomitant with a transient electronic wave function delocalization throughout the two chromophores. This is shown in Figure $4 \mathrm{~b}$, where the localization of the electronic transition density at the time of the effective $S_{2} \rightarrow S_{1}$ transition is displayed.

While the complete electronic relaxation takes place in about $20 \mathrm{fs}$ at $10 \mathrm{~K}$ (Figure $2 \mathrm{a}$ ), this process lasts more than $200 \mathrm{fs}$ to be completed at $300 \mathrm{~K}$ (Figure $2 \mathrm{~b}$ ). In principle, two possible processes should be taken into account. On one hand, we can consider a relaxation pathway that involves energy transfer between monomers through internal conversion between $S_{1}$ and $S_{2}$ states (interchromophore dynamics) occurring due to weak electronic coupling between chromophores. On the other hand, the relaxation of the electronic energy can take place within the same chain that has been initially excited (intrachromophore vibrational relaxation). 

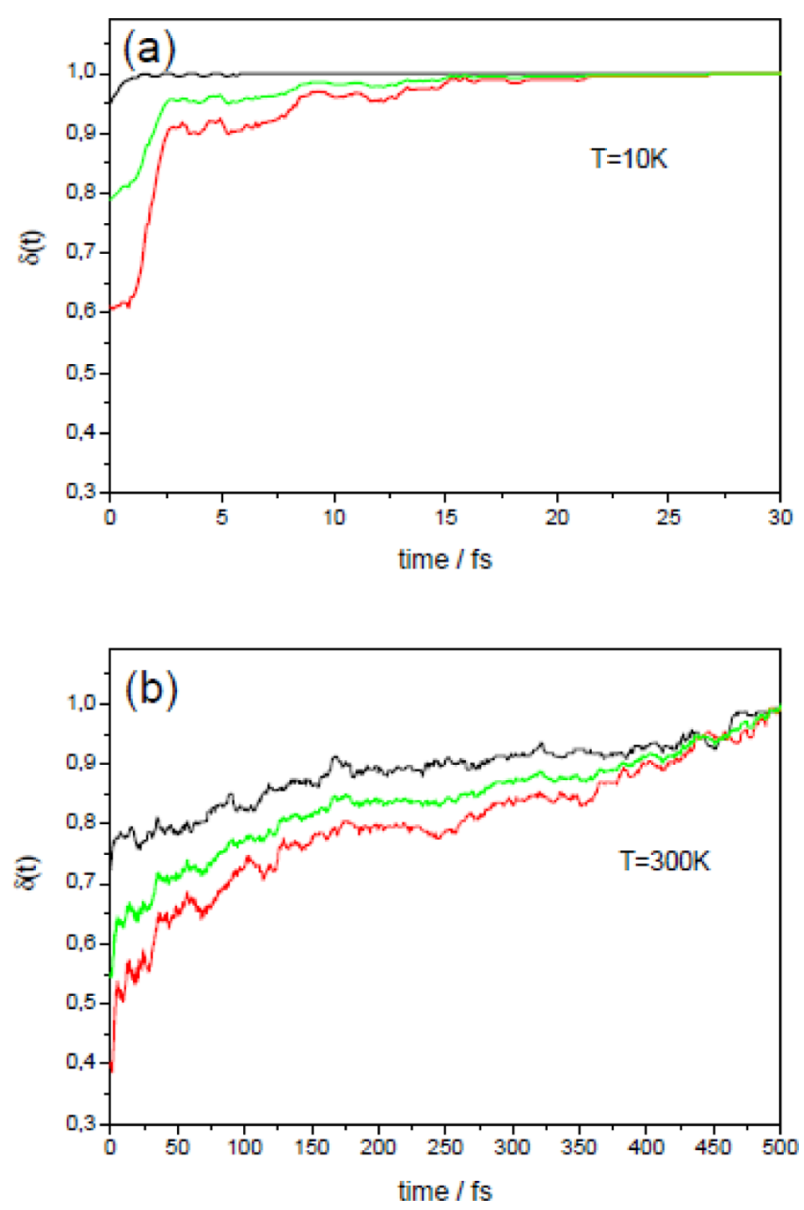

Figure 6. Time evolution of the overlap between the current and final transition densities averaged over the trajectories starting on $S_{1}$ (black line), averaged over the trajectories starting on $S_{2}$ (red line), and averaged over all trajectories (green line).

In order to discriminate between these pathways, we evaluate the time evolution of the overlap between the current (i.e., at time $t$ ) and final transition densities

$$
\delta(t)=\sum_{\mathrm{n}, \mathrm{m}}\left(\rho_{\mathrm{nm}}^{g \alpha}(t)\right)^{2} \cdot\left(\rho_{\mathrm{nm}}^{g S_{1}}\left(t_{\infty}\right)\right)^{2}
$$

with $t_{\infty}$ being 500 fs (i.e., the final time of the NA-ESMD trajectories), and index $\alpha$ indicates the current electronic excited state at time $t$. Since over 500 fs all trajectories have finished in the lowest $S_{1}$ excited state, eq 6 can separate distinct bundles of trajectories according to the initial value of $\delta(0)$. The left panels in Figure 5 display the time evolution of $\delta(t)$ numerically averaged over trajectories with $\delta(0)>0.5$, averaged over trajectories with $\delta(0)<0.5$, and over all trajectories. Due to weak electronic coupling $S_{1}$ and $S_{2}$ states barely mix into a quantum superposition (i.e., in a Frenkel exciton sense). Subsequently, the sets of trajectories with $\delta(0)>0.5$ and $\delta(0)<$ 0.5 actually present averages $\delta(0) \approx 1$ and $\delta(0) \approx 0$, respectively. Considering that the final transition density, corresponding to the emission state, is completely localized on a single chain of the composite molecule, values of $\delta(0) \approx 0$ correspond to trajectories that absorb in the opposite chain that emit (interchromophore channel), and values of $\delta(0) \approx 1$ indicate trajectories where the excitation and emission take place from the same chain (intrachromophore channel). These two sets of trajectories are clearly observed at both $10 \mathrm{~K}$
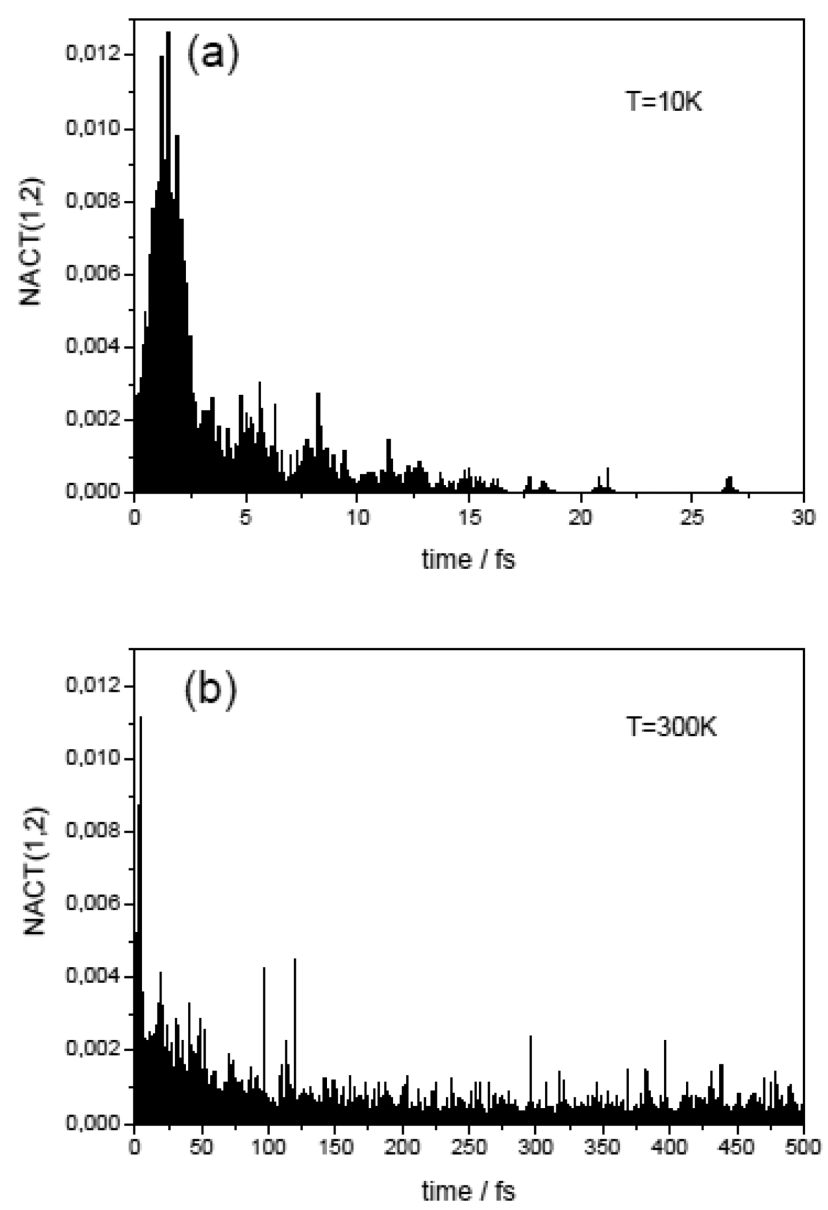

Figure 7. Absolute value of $\operatorname{NACT}(1,2)$ averaged over all trajectories: (a) at $10 \mathrm{~K}$ and (b) at $300 \mathrm{~K}$.

(Figure 5a) and $300 \mathrm{~K}$ (Figure 5b). Therefore, the photoexcited dynamics of the spiro-linked polyfluorene follows two distinct pathways, as described above. The relative contributions of the different pathways vary substantially with temperature. The right panels in Figure 5 illustrate the wavepacket dynamics, which is represented as the evolution in time of the distribution of the values of $\delta(t)$ at $10 \mathrm{~K}$ and 300 $\mathrm{K}$. While only $20 \%$ of all the trajectories at $10 \mathrm{~K}$ follow the interchromophore channel, that is, start with initial transition densities localized on the different branch than the final one $(\delta(0) \approx 0)$, these trajectories represent more than a $46 \%$ fraction at $300 \mathrm{~K}$. Moreover, no intermediate states with values of $\delta(0) \approx 0.5$, representing an electronic excitation significantly delocalized between both chains, have be detected throughout the NA-ESMD simulations.

In principle, one can propose that the initial excitation to the $\mathrm{S}_{2}$ state is directly related to the interchromophore channel involving the electronic energy transfer between the monomer chains, while the excitation to the $S_{1}$ state is associated with the intrachromophore relaxation of the electronic energy in a single chain. To test this hypothesis, the average values of $\delta(t)$ have been analyzed separately for trajectories starting on both states. The results are shown in Figure 6. We start our analysis with the low temperature $10 \mathrm{~K}$ results (Figure 6a). The average $\delta(\mathrm{t}$ ) $\approx 1$ throughout the entire photoinduced dynamics for trajectories starting at the $S_{1}$ state indicates that these trajectories follow a relaxation pathway on the same chain that has been initially excited. On the other hand, trajectories 

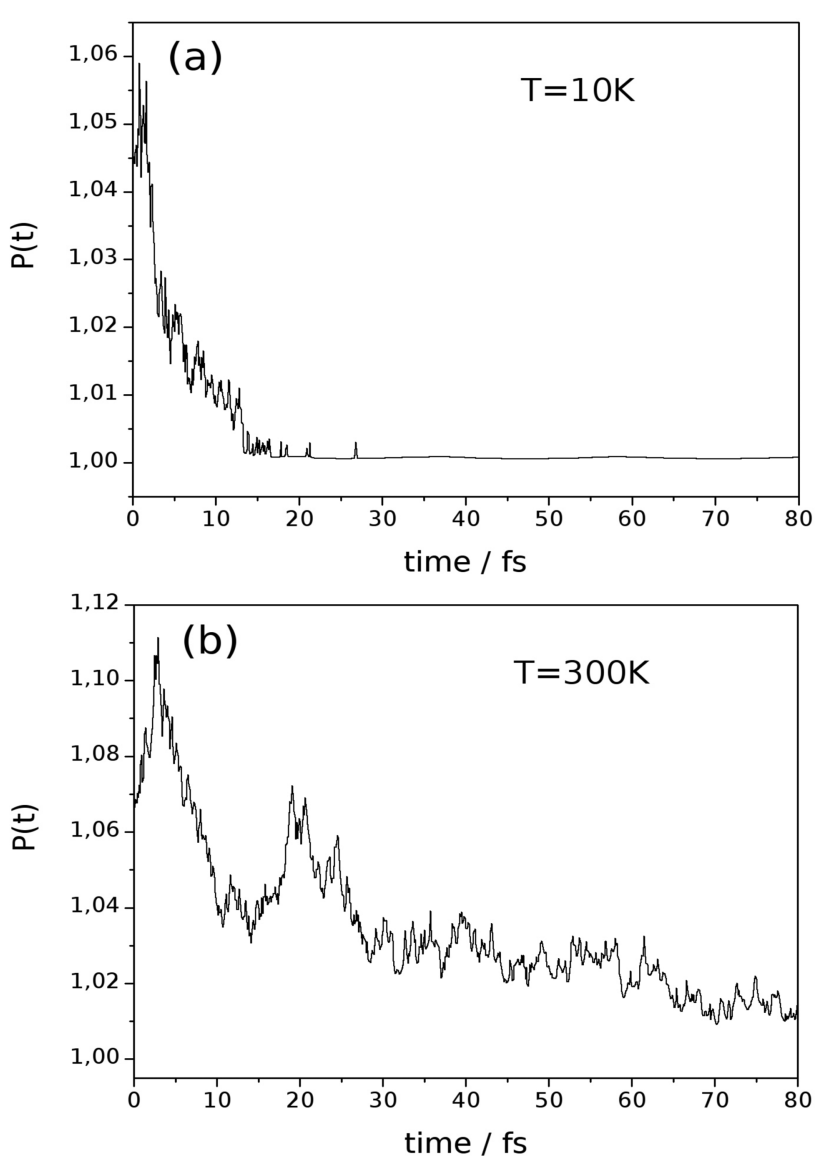

Figure 8. Time-dependence of the average monomer participation number: (a) at $10 \mathrm{~K}$ and (b) at $300 \mathrm{~K}$.

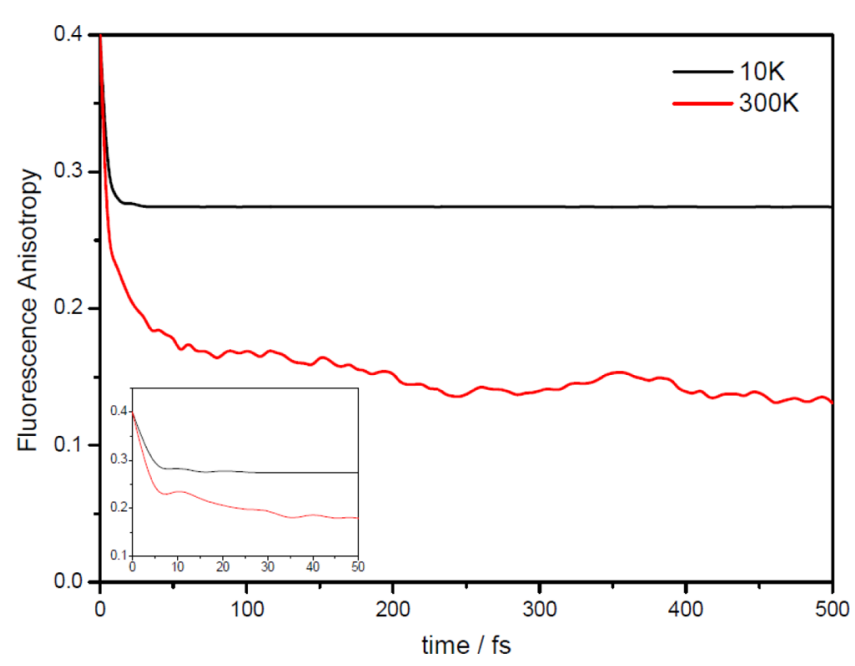

Figure 9. Calculated time-resolved fluorescence anisotropy averaged over all trajectories.

starting at the $S_{2}$ state present an average value of $\delta(0) \approx 0.6$. According to results shown in Figure 5a, no trajectories are observed with values of $\delta(0)$ different from $\sim 1$ or $\sim 0$. Therefore, we can conclude that only $\sim 40 \%$ of trajectories initially excited to $S_{2}$ undergo internal conversion with electronic energy transfer between chains. The other $60 \%$ of trajectories starting at $S_{2}$ experience a vibrational relaxation path involving only one chain. Since all the trajectories finish on $S_{1}$, at least one trivial unavoided crossing between $S_{1}$ and $S_{2}$ must take place where the system follows the "diabatic pathway" and the energy order of the corresponding diabatic states is inverted. ${ }^{70}$ Because of such crossings, the assignment of the adiabatic states based on the energy-ordering criterion at each time during dynamics becomes useless. The situation is even more pronounced at $300 \mathrm{~K}$ (Figure 6b) where, according to the average values of $\delta(0)$, more than $60 \%$ of trajectories starting on $\mathrm{S}_{2}$ are effectively changing a chain. Furthermore, either trajectory starting on $S_{1}$ or $S_{2}$ experiences trivial unavoided crossings during longer times throughout the relaxation process.

The existence of trivial unavoided crossings is a consequence of the weak interaction between chains. These crossings are expected during excited state dynamics in extended conjugated molecules, where noninteracting states localized on different moieties of a molecule share a common energy range. This is particularly the case of spiro-linked conjugated molecules. In Figure 7, the time dependence of the nonadiabatic coupling terms (NACTs, ${ }^{57}$ see eq 3 ) between $S_{1}$ and $S_{2}$ is depicted. The values of NACT for trajectories at $10 \mathrm{~K}$ (Figure $7 \mathrm{a}$ ) reveal a negligible coupling between the chains at times greater than $\sim 20$ fs. Furthermore, the peak of NACT distribution correlates with the time at which the conical intersection seam is reached (see Figure 3a). In contrast, a very weak coupling persists during all the dynamics at $300 \mathrm{~K}$ (Figure $7 \mathrm{~b}$ ), which is attributed to the thermally induced conformational motions bringing states to the resonant condition multiple times. This explains the major contribution of the interchromophore channel involving the electronic energy transfer between chains at $300 \mathrm{~K}$ as observed in Figure $5 \mathrm{~b}$.

At this point it is important to stress our finding that, despite the slower vibrational relaxation, a more efficient ultrafast electronic relaxation is observed at low temperature. We show that this behavior can be attributed to the observed temperature dependence in the relative contributions of inter- and intrachromophore relaxation channels. Thermally induced conformational motions keep electronic states coupled for longer times and, therefore, more events of interchromophore energy exchange take place.

In order to analyze how the weak interaction between monomers leads to a minor mix between states, in Figure 8 we represent the time-dependence of the average monomer participation number calculated with eq 5. Only a small delocalization can be observed at short times of the lowtemperature dynamics. In agreement with the lack of coupling after $20 \mathrm{fs}$, as shown in Figure 7, the excited state wave function becomes completely confined on a single monomer after that time. As expected, only a slightly larger mixture that persists even at long times of the dynamics is observed at $300 \mathrm{~K}$ (Figure $8 \mathrm{~b})$. Nevertheless, either at $10 \mathrm{~K}$ (Figure $8 \mathrm{a}$ ) or at $300 \mathrm{~K}$ (Figure $8 \mathrm{~b}$ ) the average value of $\mathbf{P}(t)$ reaches its final value of 1 , confirming that the emission comes from the localized (selftrapped) excitons. That is, both pathways ultimately result in the self-trapping of the excitation on a single chain.

Spectroscopically, the change of localization of the exciton during the photoexcited dynamics can be probed by timeresolved fluorescence anisotropy. To simulate the latter, we calculate the autocorrelation function of the absorption dipole moment of the chromophore at time zero, $\vec{\mu}_{\mathrm{A}}(t=0)$, and its emission dipole moment at time $t, \vec{\mu}_{\mathrm{E}}(t):{ }^{91}$

$$
r(t)=\frac{2}{5}\left\langle P_{2}\left\{\vec{\mu}_{\mathrm{A}}(0) \cdot \vec{\mu}_{\mathrm{E}}(t)\right\}\right\rangle
$$



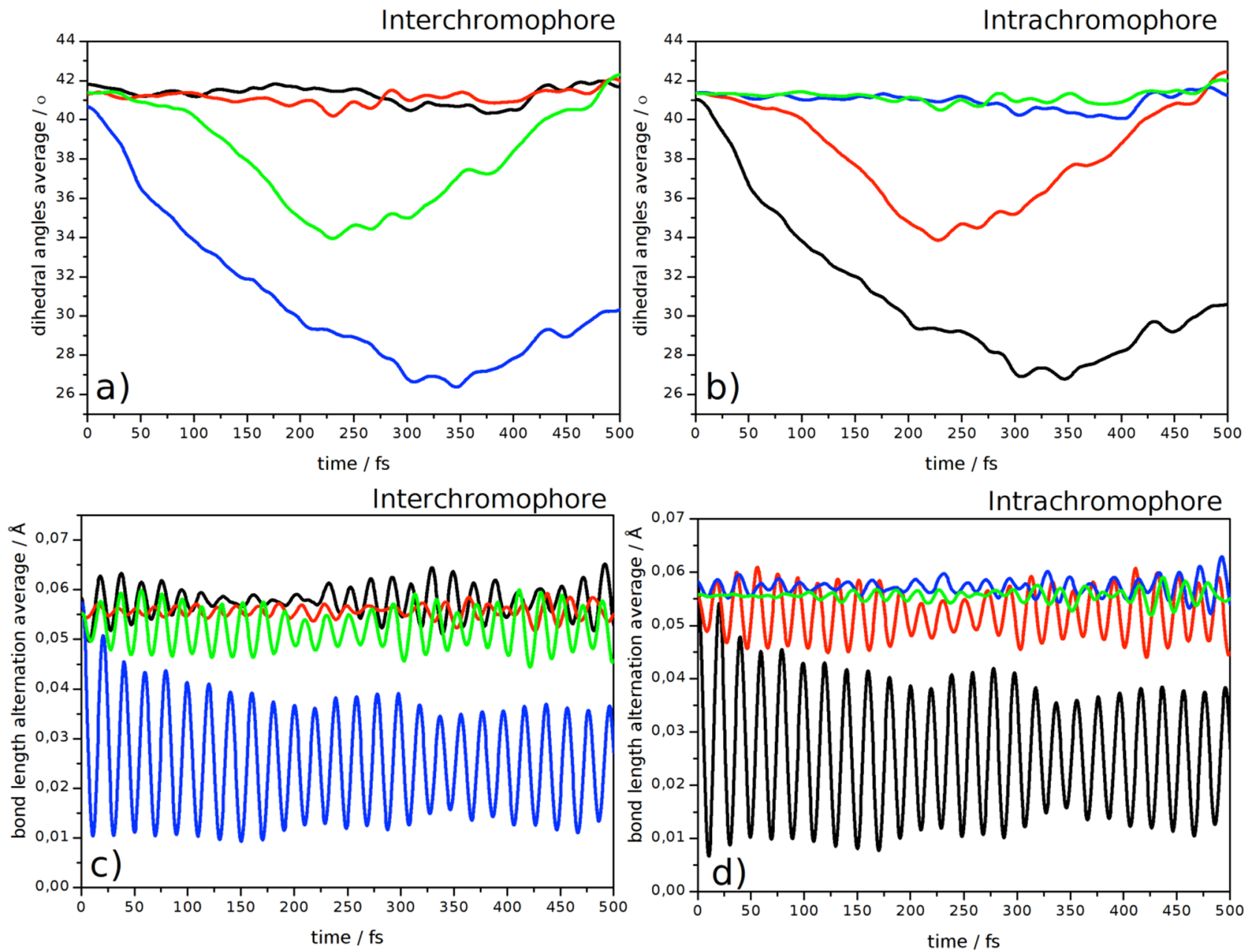

Figure 10. Time evolution of the dihedral angles and bond length alternations averaged over the NA-ESMD trajectories at $10 \mathrm{~K}$ with (a and c) the initial transition density localized on the different branch as the final one $(\delta(0)<0.5)$ and $(\mathrm{b}$ and d) initial transition density localized on the same branch as the final one $(\delta(0)>0.5)$. The dihedral angles and bond length alternations are defined as central, connecting units $2-3$ and $3-4$ in the monomer that absorb (black), and do not absorb (blue); and peripheral, connecting units 1-2 and 4-5 in the monomer that absorb (red) and do not absorb (green).

where $P_{2}(x)=(1 / 2)\left(3 x^{2}-1\right)$ is the second-order Legendre polynomial and the angular brackets denote the average over all the trajectories. Figure 9 shows variation of calculated $r(t)$. Considering the ultrafast time scale of the relaxation process under study, we can assume that the energy transfer between monomers is the only source of fluorescence depolarization and we neglect the rotational diffusion typically occurring at much longer time-scales. As has been previously discussed, $\vec{\mu}_{\mathrm{A}}(0)$ is localized on one of the two chains and the internal conversion leads to the energy transfer between chains and, therefore, a rotation of $\sim 90^{\circ}$ of $\vec{\mu}_{\mathrm{E}}(t)$. Higher energy transfer rate between chains observed in the higher temperature simulations ensures faster fluorescence anisotropy decay compared to the low temperature results. Furthermore, at $10 \mathrm{~K}$ no further decay is observed at times longer than $20 \mathrm{fs}$, due to lack of coupling between $S_{1}$ and $S_{2}$ states. In contrast, a persistent slow decay is present throughout the entire dynamics at $300 \mathrm{~K}$.

Finally, to analyze molecular conformations during excited state dynamics, we follow the time evolution of the dihedral angles and bond length alternations defined at central (connecting fluorene units 2-3, and 3-4) and peripheral (connecting units 1-2, and 4-5) parts of the oligomer; see Figure 1a. Dihedral angles are defined as the deviation from $180^{\circ}$ of the torsional angle between the neighboring fluorene units due to single bond rotation. The bond length alternation is defined as a difference in the bond length $d_{2}-\left(d_{1}+d_{3}\right) / 2$ as shown in Figure 1a. ${ }^{56}$ Small values of dihedral angles and bond length alternations are subsequently associated with planar structures promoting the extent of the $\pi$-conjugation throughout the two corresponding fluorene units. ${ }^{56}$ Both parameters have been analyzed separately for trajectories that follow the interchromophore channel, and trajectories that follow the intrachromophore channel. The results are displayed in Figures 10 and 11 for simulations at 10 and $300 \mathrm{~K}$, respectively. In all cases, the initial dihedral angle is $\sim 41^{\circ}$, being close to the equilibrium geometry value. ${ }^{56}$ This reflects strong steric interactions between the neighboring units in the ground state. Similarly, large initial value of bond length alternation averaged over the trajectories corresponds to the ground state geometry as well.

First let us analyze variation of these parameters during dynamics for trajectories that follow the intrachromophore channel at $10 \mathrm{~K}$ (Figure 10b,d). After photoexcitation, we observe reduction of dihedral angles and bond length alternations on the chain that absorb (and emit in this case), being more pronounced for central than for peripheral bonds. 

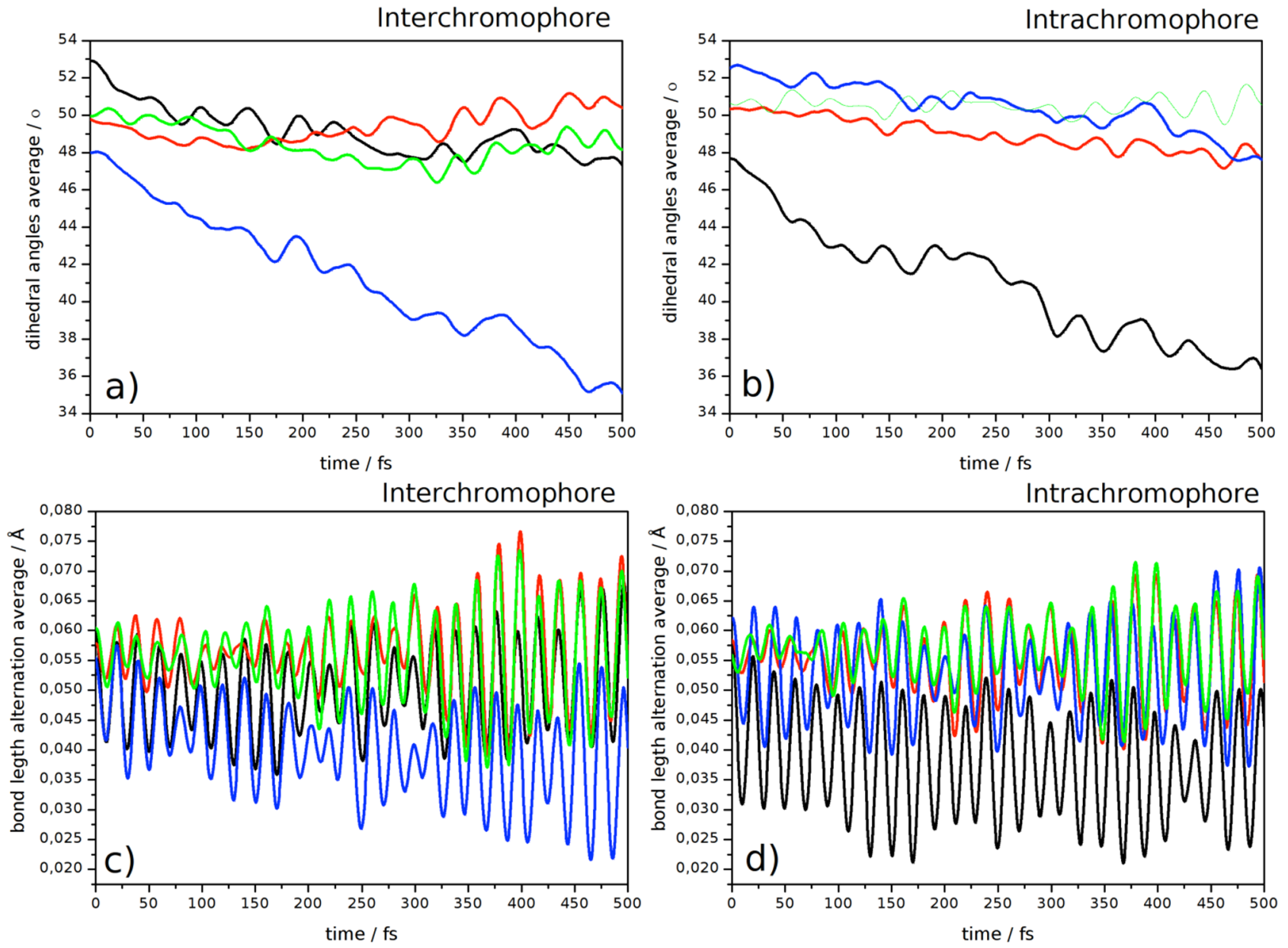

Figure 11. Same as Figure 10 but at $300 \mathrm{~K}$.

This localized pattern is a characteristic signature of the dynamical exciton self-trapping, that is, confinement of the excitation on a short conjugated segment due to vibrational relaxation. ${ }^{92}$ In contrast, vanishing geometrical changes are observed for the chain that is never excited and is not participating in the dynamics. A very similar picture is observed for trajectories that follow the interchromophore channel at 10 $\mathrm{K}$ (Figure 10a,c). However, here, varying dihedral angles and bond length alternations are the ones corresponding to the chain that do not absorb (but emit). The difference can be explained by considering that the energy transfer event between chains mostly takes place during the very first 5 fs of the dynamics; after that, the exciton is localized on the chain that finally emits. Finally, Figure 11 shows more complicated interplay of the photoexcitation dynamics and the concomitant nuclear motions at $300 \mathrm{~K}$. The higher thermal noise leads to smaller effective changes in the central dihedral angles and bond length alternations of the chains that localize the exciton during the relaxation dynamics.

\section{CONCLUSIONS}

We have used the NA-ESMD framework to simulate photoexcited dynamics of spiro-linked conjugated polyfluorene that represents a model of weakly interacting conjugated molecular aggregate where the resonant interchromophore energy transfer may occur. The negligible coupling between the monomer chains results in the absorption band composed of equal contributions of the two lowest $S_{1}$ and $S_{2}$ electronic excited states. Initially, each of these states is completely localized on each of the two oligomers. While the initial wavepacket created by laser excitation has similar contributions from $S_{1}$ and $S_{2}$ states, an efficient ultrafast localization of all the electronic population in the lowest $S_{1}$ state is observed during nonadiabatic excited state dynamics. As a result of this relaxation process, an emission spectrum of the PF homodimer is similar to that of the individual monomer.

Two distinct pathways contribute to the electronic relaxation process within 500 fs of dynamical simulations. The interchromophore channel involves an internal conversion of the two lowest excited states, and therefore, the electronic energy transfer between the monomer chains followed by concurrent vibrational relaxation. In contrast, the intrachromophore path implies the relaxation of the electronic energy in the same chain that has been initially excited, a process similar to a relaxation of the individual monomer.

The spiro-linked conjugated PF represents a case of extended conjugated molecule where noninteracting states localized in different moieties of a molecule share a common energy range. As a consequence of negligible coupling between chains, trivial unvoided crossings between $S_{1}$ and $S_{2}$ are common and require special numerical algorithms. ${ }^{71,93}$ To analyze observed electronic dynamics, we followed spatial distribution of the electronic transition density since assignment in terms of adiabatic states determined by energy ordering is impractical. This allowed us to discriminate between families of trajectories pursuing the inter- and intrachromophore relaxation channels. 
The relative contributions of these pathways vary substantially with the temperature: about $50 \%$ and $20 \%$ of population make use of interchromophore energy transfer at room $(T=300 \mathrm{~K})$ and low $(T=10 \mathrm{~K})$ temperatures, respectively. Notably, at low temperature the energy transfer events are occurring within the first $20 \mathrm{fs}$ of dynamics before vibrational relaxation takes place. In contrast, at room temperature the former are observed over 100 fs time scales due to thermal fluctuations by bringing states back to the resonant conditions. During such ultrafast photoexcited dynamics in the dimer, nonlinear electronic excitations such as solitons may also appear due to degeneracy of the lowest excitonic state, which have been extensively explored in other systems with quasi-degenerate states such as polyacetylene. ${ }^{94,95}$ Our simulations provide all necessary dynamical information for previously developed theoretical models ${ }^{95,96}$ for nonlinear excitations.

The final vibronically relaxed state is a self-trapped excitation on a single chain characterized by planarization of the chain and reduced bond-length alternations, while the other chain remains unchanged. ${ }^{56}$ The latter quantities characterized nuclear motions coupled to the electronic dynamics, which contribute to vibrational relaxation along the excited state potential energy surface. This distinct vibrational dynamics lifts the initial degeneracy of the two lowest electronic excited states.

To conclude, presented nonadiabatic dynamics simulations provide complete atomistic and time-resolved information on electronic and vibrational relaxation processes, providing an adequate description of the resonant energy transfer mechanism. In particular, our modeling suggests specific spectroscopic probes (i.e., time-resolved fluorescence anisotropy) that may be used to distinguish the inter- and intrachromophore relaxation channels in experiments to be directly compared to theoretical calculations. Thus, our modeling is expected to have useful implications for future experiments and design of efficient conjugated molecular fluorophores.

\section{AUTHOR INFORMATION}

\section{Corresponding Author}

*E-mail: sfalberti@gmail.com. Telephone : 0054-221-4723745.

\section{Notes}

The authors declare no competing financial interest.

\section{ACKNOWLEDGMENTS}

This work was partially supported by CONICET, UNQ, ANPCyT (PICT-2010-2375) and the U.S. Department of Energy and Los Alamos LDRD funds. Los Alamos National Laboratory is operated by Los Alamos National Security, LLC, for the National Nuclear Security Administration of the U.S. Department of Energy under contract DE-AC52-06NA25396. We acknowledge support of Center for Integrated Nanotechnology (CINT) and Center for Nonlinear Studies (CNLS) at LANL.

\section{REFERENCES}

(1) Kraft, A.; Grimsdale, A. C.; Holmes, A. B. Electroluminescent Conjugated Polymers-Seeing Polymers in a New Light. Angew. Chem., Int. Ed. 1998, 37, 402-428.

(2) Lemmer, U. Stimulated Emission and Lasing in Conjugated Polymers. Polym. Adv. Technol. 1998, 9, 476-487.

(3) Friend, R. H.; Gymer, R. W.; Holmes, A. B.; Burroughes, J. H.; Marks, R. N.; Taliani, C.; Bradley, D. D. C.; Dos Santos, D. A.; Brédas, J. L.; Lögdlund, M.; et al. Electroluminescence in Conjugated Polymers. Nature 1999, 397, 121-128.
(4) Kim, D. Y.; Cho, H. N.; Kim, C. Y. Blue Light Emitting Polymers. Prog. Polym. Sci. 2000, 25, 1089-1139.

(5) Chen, P.; Yang, G.; Liu, T.; Li, T.; Wang, M.; Huang, W. Optimization of Opto-electronic Property and Device Efficiency of Polyfluorenes by Tuning Structure and Morphology. Polym. Int. 2006, 55, 473-490.

(6) Alsalhi, M. S.; Alam, J.; Dass, L. A.; Raja, M. Recent Advances in Conjugated Polymers for Light Emitting Devices. Int. J. Mol. Sci. 2011, $12,2036-2054$.

(7) Xu, Y.; Zhang, F.; Feng, X. Patterning of Conjugated Polymers for Organic Optoelectronic Devices. Small 2011, 7, 1338-1360.

(8) Tang, C.; Liu, X.-D.; Liu, F.; Wang, X.-L.; Xu, H.; Huang, W. Recent Progress in Polymer White Light-Emitting Materials and Devices. Macromol. Chem. Phys. 2013, 214, 314-342.

(9) Friend, R. H. Conjugated Polymers. New Materials for Optoelectronic Devices. Pure Appl. Chem. 2001, 73, 425-430.

(10) Lu, S.; Fan, Q.-L.; Xiao, Y.; Chua, S.-J.; Huang, W. Synthesis and Characterization of a Novel Conjugated Polymer Containing PPV Oligomer and Fluorene. Thin Solid Films 2002, 417, 215-220.

(11) Wong, K. T.; Chen, R. T.; Fang, F. C.; Wu, C. C.; Lin, Y. T. 4,5Diazafluorene-Incorporated Ter (9, 9-diarylfluorene): A Novel Molecular Doping Strategy for Improving the Electron Injection Property of a Highly Efficient OLED Blue Emitter. Org. Lett. 2005, 7, 1979-1982.

(12) Heeger, A. J. Light Emission from Semiconductiong Polymers: Light-emitting Diodes, Light-emitting Electrochemical Cells, Lasers and White Light for the Future. Solid State Commun. 1998, 107, 673679.

(13) Sheng, C. X.; Singh, S.; Gambetta, A.; Drori, T.; Tong, M.; Tretiak, S.; Vardeny, Z. V. Ultrafast Intersystem-crossing in Platinum Containing $\pi$-conjugated Polymers with Tunable Spin-orbit Coupling. Sci. Rep. 2013, 3, 2653-2659.

(14) Zhen, C.-G.; Dai, Y.-F.; Zeng, W.-J.; Ma, Z.; Chen, Z.-K.; Kieffer, J. Achieving Highly Efficient Fluorescent Blue Organic Lightemitting Diodes Through Optimizing Molecular Structures and Device Configuration. Adv. Funct. Mater. 2011, 21, 699-707.

(15) Sun, Y.; Giebink, N. C.; Kanno, H.; Ma, B.; Thompson, M. E.; Forrest, S. R. Management of Singlet and Triplet Excitons for Efficient White Organic Light-emitting Devices. Nature 2006, 440, 908-912.

(16) Bernius, M.; Inbasekaran, M.; Woo, E.; Wu, W.; Wujkowski, L. Light-emitting Diodes Based on fluorene Polymers. Thin Solid Films 2000, 363, 55-57.

(17) Bernius, M. T.; Inbasekaran, M.; O’Brien, J.; Wu, W. Progress with Light-emitting Polymers. Adv. Mater. 2000, 12, 1737-1750.

(18) Zeng, G.; Yu, W.-L.; Chua, S.-J.; Huang, W. Spectral and Thermal Spectral Stability Study for Fluorene-Based Conjugated Polymers. Macromolecules 2002, 35, 6907-6914.

(19) Scherf, B. U.; List, E. J. W. Semiconducting PolyfluorenesTowards Reliable Structure and Property Relationships. Adv. Mater. 2002, 14, 477-487.

(20) Wallace, J. U.; Chen, S. H. Fluorene-Based Conjugated Oligomers for Organic Photonics and Electronics. Adv. Polym. Sci. 2008, 212, 145-186.

(21) Chen, J.-C.; Lee, T.-S.; Lin, C.-H. Syntheses of Ladder-type Oligonaphthalene Derivatives and Their Photophysical and Electrochemical Properties. Chemistry 2008, 14, 2777-2787.

(22) Becker, S.; Ego, C.; Grimsdale, A. C.; List, E. J. W.; Marsitzky, D.; Pogantsch, A.; Setayesh, S.; Leising, G.; Müllen, K. Optimisation of Polyfluorenes for Light Emitting Applications. Synth. Met. 2002, 125, $73-80$.

(23) Müller, C. D.; Falcou, A.; Reckefuss, N.; Rojahn, M.; Wiederhirn, V.; Rudati, P.; Frohne, H.; Nuyken, O.; Becker, H.; Meerholz, K. Multi-colour Organic Light-emitting Displays by Solution Processing. Nature 2003, 421, 829-833.

(24) Monkman, A.; Rothe, C.; King, S.; Dias, F. Polyfluorene Photophysics. Adv. Polym. Sci. 2008, 212, 187-225.

(25) Knaapila, M.; Monkman, A. P. Methods for Controlling Structure and Photophysical Properties in Polyfluorene Solutions and Gels. Adv. Mater. 2013, 25, 1090-1108. 
(26) Zeng, G.; Chua, S.-J.; Huang, W. Influence of Donor and Acceptor Substituents on the Electronic Characteristics of Poly(fluorene-phenylene). Thin Solid Films 2002, 417, 194-197.

(27) Liu, B.; Yu, W.-L.; Lai, Y.-H.; Huang, W. Blue-Light-Emitting Cationic Water-Soluble Polyfluorene Derivatives with Tunable Quaternization Degree. Macromolecules 2002, 35, 4975-4982.

(28) Miteva, T.; Meisel, A.; Knoll, W.; Nothofer, H. G.; Scherf, U.; Müller, D. C.; Meerholz, K.; Yasuda, A.; Neher, D. Improving the Performance of Polyfluorene-Based Organic Light-emitting Diodes via End-capping. Adv. Mater. 2001, 13, 565-570.

(29) Ling, Q. D.; Kang, E. T.; Neoh, K. G.; Huang, W. Synthesis and Nearly Monochromatic Photoluminescence Properties of Conjugated Copolymers Containing Fluorene and Rare Earth Complexes. Macromolecules 2003, 36, 6995-7003.

(30) Xie, L.-H.; Yin, C.-R.; Lai, W.-Y.; Fan, Q.-L.; Huang, W. Polyfluorene-based Semiconductors Combined with Various Periodic Table Elements for Organic Electronics. Prog. Polym. Sci. 2012, 37, $1192-1264$.

(31) Liu, B.; Yu, W.-L.; Pei, J.; Liu, S.-Y.; Lai, Y.-H.; Huang, W. Design and Synthesis of Bipyridyl-Containing Conjugated Polymers: Effects of Polymer Rigidity on Metal Ion Sensing. Macromolecules 2001, 34, 7932-7940.

(32) Grell, M.; Bradley, D. D. C.; Inbasekaran, M.; Woo, E. P. A Glass-forming Conjugated Main-chain Liquid Crystal Polymer for Polarized Electroluminescence Applications. Adv. Mater. 1997, 9, 798-802.

(33) Grell, M.; Bradley, D. D. C. .; Long, X.; Chamberlain, T.; Inbasekaran, M.; Woo, E.; Soliman, M. Chain Geometry, Solution Aggregation and Enhanced Dichroism in the Liquid-crystalline Conjugated Polymer Poly(9,9-dioctylfluorene). Acta Polym. 1998, 49, 439-444.

(34) Gong, X. .; Iyer, P. K.; Moses, D.; Bazan, G. C.; Heeger, A. J.; Xiao, S. S. Stabilized Blue Emission from Polyfluorene-Based LightEmitting Diodes: Elimination of Fluorenone Defects. Adv. Funct. Mater. 2003, 13, 325-330.

(35) List, E. J. W.; Guentner, R.; Scanducci De Freitas, P.; Scherf, U. The Effect of Keto Defect Sites on the Emission Properties of Polyfluorene-type Materials. Adv. Mater. 2002, 14, 374-378.

(36) Klärner, G.; Lee, J.-I.; Davey, M. H.; Miller, R. D. Exciton Migration and Trapping in Copolymers Based on Dialkylfluorenes. Adv. Mater. 1999, 11, 115-119.

(37) Lu, H.-H.; Liu, C.-Y.; Jen, T.-H.; Liao, J.-L.; Tseng, H.-E.; Huang, C.-W.; Hung, M.-C.; Chen, S.-A. Excimer Formation by Electric Field Induction and Side Chain Motion Assistance in Polyfluorenes. Macromolecules 2005, 38, 10829-10835.

(38) Sims, M.; Bradley, D. D. C.; Ariu, M.; Koeberg, M.; Asimakis, A.; Grell, M.; Lidzey, D. G. Understanding the Origin of the $535 \mathrm{Nm}$ Emission Band in Oxidized Poly(9,9-dioctylfluorene): The Essential Role of Inter-Chain/Inter-Segment Interactions. Adv. Funct. Mater. 2004, 14, 765-781.

(39) Becker, K.; Lupton, J. M.; Feldmann, J.; Nehls, B. S.; Galbrecht, F.; Gao, D. Q.; Scherf, U. On-chain Fluorenone Defect Emission from Single Polyfluorene Molecules in the Absence of Intermolecular Interactions. Adv. Funct. Mater. 2006, 16, 364-370.

(40) Weinfurtner, K.-H.; Fujikawa, H.; Tokito, S.; Taga, Y. Highly Efficient Pure Blue Electroluminescence from Polyfluorene: Influence of the Molecular Weight Distribution on the Aggregation Tendency. Appl. Phys. Lett. 2000, 76, 2502-2504.

(41) Nguyen, T.-Q.; Martini, I. B.; Liu, J.; Schwartz, B. J. Controlling Interchain Interactions in Conjugated Polymers: The Effects of Chain Morphology on Exciton-exciton Annihilation and Aggregation in MEH-PPV Films. J. Phys. Chem. B 2000, 104, 237-255.

(42) Kreyenschmidt, M.; Klaerner, G.; Fuhrer, T.; Ashenhurst, J.; Karg, S.; Chen, W. D.; Lee, V. Y.; Scott, J. C.; Miller, R. D. Thermally Stable Blue-Light-Emitting Copolymers of Poly (Alkylfluorene). Macromolecules 1998, 31, 1099-1103.

(43) Klärner, G.; Davey, M. H.; Chen, W.-D.; Scott, J. C.; Miller, R. D. Colorfast Blue-Light-Emitting Random Copolymers Derived from Di-n-hexylfluorene and Anthracene. Adv. Mater. 1998, 10, 993-997.
(44) Andersson, M. R.; Thomas, O.; Mammo, W.; Svensson, M.; Theanderc, M.; Ingana, O. Substituted Polythiophenes Designed for Optoelectronic Devices and Conductors. J. Mater. Chem. 1999, 9, 1933-1940.

(45) Theander, M.; Inganäs, O.; Mammo, W.; Olinga, T.; Svensson, M.; Andersson, M. R. Photophysics of Substituted Polythiophenes. J. Phys. Chem. B 1999, 103, 7771-7780.

(46) Yu, W.-L.; Pei, J.; Huang, W.; Heeger, a. J. Spiro-Functionalized Polyfluorene Derivatives as Blue Light-Emitting Materials. Adv. Mater. 2000, 12, 828-831.

(47) Marsitzky, D.; Vestberg, R.; Blainey, P.; Tang, B. T.; Hawker, C. J.; Carter, K. R. Self-Encapsulation of Poly-2,7-fluorenes in a Dendrimer Matrix. J. Am. Chem. Soc. 2001, 123, 6965-6972.

(48) Setayesh, S.; Grimsdale, A. C.; Weil, T.; Enkelmann, V.; Müllen, K.; Meghdadi, F.; List, E. J. W.; Leising, G. Polyfluorenes with Polyphenylene Dendron Side Chains: Toward Non-Aggregating, Light-Emitting Polymers. J. Am. Chem. Soc. 2001, 123, 946-953.

(49) Wu, F.-I.; Dodda, R.; Reddy, D. S.; Shu, C.-F. Synthesis and Characterization of Spiro-linked Poly(terfluorene): a Blue-emitting Polymer with Controlled Conjugated Length. J. Mater.Chem. 2002, 12, 2893-2897.

(50) Wu, C.-I.; Lee, G.-R.; Lin, C.-T.; Chen, Y.-H.; Hong, Y.-H.; Liu, W.-G.; Wu, C.-C.; Wong, K.-T.; Chao, T.-C. Influences of Substitution on Electronic Structures of Oligofluorenes. Appl. Phys. Lett. 2005, 87, 242107-24219.

(51) Pudzich, R.; Fuhrmann-Lieker, T.; Salbeck, J. Spiro Compounds for Organic Electroluminescence and Related Applications. Adv. Polym. Sci. 2006, 199, 83-142.

(52) Saragi, T. P. I.; Spehr, T.; Siebert, A.; Fuhrmann-Lieker, T.; Salbeck, J. Spiro Compounds for Organic Optoelectronics. Chem. Rev. 2007, 107, 1011-1065.

(53) Yang, Z.-D.; Feng, J.-K.; Ren, A.-M. Spiro-linked Oligofluorenes and Derivatives: Molecular Design and Theoretical Study of One- and Two-photon Absorption Properties. Chem. Phys. Lett. 2008, 461, 915

(54) Kanibolotsky, A. L.; Perepichka, I. F.; Skabara, P. J. Star-shaped Pi-conjugated Oligomers and Their Applications in Organic Electronics and Photonics. Chem. Soc. Rev. 2010, 39, 2695-2728.

(55) Komino, T.; Nomura, H.; Yahiro, M.; Endo, K.; Adachi, C. Dependence of the Amplified Spontaneous Emission Threshold in Spirofluorene Thin Films on Molecular Orientation. J. Phys. Chem. C 2011, 115, 19890-19896.

(56) Franco, I.; Tretiak, S. Electron-vibrational Dynamics of Photoexcited Polyfluorenes. J. Am. Chem. Soc. 2004, 126, 1213012140.

(57) Nelson, T.; Fernandez-Aberti, S.; Chernyak, V.; Roitberg, A. E.; Tretiak, S. Nonadiabatic Excited-state Molecular Dynamics Modeling of Photoinduced Dynamics in Conjugated Molecules. J. Phys. Chem. B 2011, 115, 5402-5414.

(58) Nelson, T.; Fernandez-Alberti, S.; Chernyak, V.; Roitberg, A. E.; Tretiak, S. Nonadiabatic Excited-state Molecular Dynamics: Numerical Tests of Convergence and Parameters. J. Chem. Phys. 2012, 136, 054108-054120.

(59) Nelson, T.; Fernandez-Alberti, S.; Roitberg, A. E.; Tretiak, S. Nonadiabatic Excited State Molecular Dynamics: Modeling Photophysics in Organic Conjugated Materials. Acc. Chem. Res. 2014, 47, $1155-1164$.

(60) Clark, J.; Nelson, T.; Tretiak, S.; Cirmi, G.; Lanzani, G. Femtosecond Torsional Relaxation. Nat. Phys. 2012, 8, 225-231.

(61) Fernandez-Alberti, S.; Kleiman, V. D.; Tretiak, S.; Roitberg, A. E. Nonadiabatic Molecular Dynamics Simulations of the Energy Transfer Between Building Blocks in a Phenylene Ethynylene Dendrimer. J. Phys. Chem. A 2009, 113, 7535-7542.

(62) Fernandez-Aberti, S.; Kleiman, V. D.; Tretiak, S.; Roitberg, A. E. Unidirectional Energy Transfer in Conjugated Molecules: the Crucial Role of High Frequency C (Triple) C Bonds. J. Phys. Chem. Lett. 2010, $1,2699-2704$.

(63) Fernandez-Alberti, S.; Roitberg, A. E.; Kleiman, V. D.; Nelson, T.; Tretiak, S. Shishiodoshi Unidirectional Energy Transfer Mecha- 
nism in Phenylene Ethynylene Dendrimers. J. Chem. Phys. 2012, 137, 22A526-22A535.

(64) Tretiak, S.; Saxena, A.; Martin, R. L.; Bishop, A. R. CEO/ semiempirical Calculations of UV-visible Spectra in Conjugated Molecules. Chem. Phys. Lett. 2000, 331, 561-568.

(65) Tretiak, S.; Saxena, A.; Martin, R. L.; Bishop, A. R. Photoexcited Breathers in Conjugated Polyenes: An Excited-state Molecular Dynamics Study. Proc. Natl. Acad. Sci. U.S.A. 2003, 100, 2185-2190.

(66) Worth, G. A.; Robb, M. A.; Lasorne, B. Solving the Timedependent Schrodinger Equation for Nuclear Motion in One Step: Direct Dynamics of Non-adiabatic Systems. Mol. Phys. 2008, 106, 2077-2091.

(67) Tully, J. C. Molecular Dynamics with Electronic Transitions. J. Chem. Phys. 1990, 93, 1061-1071.

(68) Hammes-Schiffer, S.; Tully, J. C. Proton Transfer in Solution: Molecular Dynamics with Quantum Transitions. J. Chem. Phys. 1994, 101, 4657-4667.

(69) Tully, J. C. Nonadiabatic Molecular Dynamics. Int. J. Quantum Chem. Symp. 1991, 309, 299-309.

(70) Fernandez-Alberti, S.; Roitberg, A. E.; Nelson, T.; Tretiak, S. Identification of Unavoided Crossings in Nonadiabatic Photoexcited Dynamics Involving Multiple Electronic States in Polyatomic Conjugated Molecules. J. Chem. Phys. 2012, 137, 14512-14521.

(71) Nelson, T.; Fernandez-alberti, S.; Roitberg, A. E.; Tretiak, S. Nonadiabatic Excited-State Molecular Dynamics: Treatment of Electronic Decoherence. J. Chem. Phys. 2013, 138, 224111-224123.

(72) Tretiak, S.; Mukamel, S. Density Matrix Analysis and Simulation of Electronic Excitations in Conjugated and Aggregated Molecules. Chem. Rev. 2002, 102, 3171-3212.

(73) Chernyak, V.; Schulz, M. F.; Mukamel, S.; Tretiak, S.; Tsiper, E. V. Krylov-space Algorithms for Time-dependent Hartree-Fock and Density Functional Computations. J. Chem. Phys. 2000, 113, 36-43.

(74) Tretiak, S.; Isborn, C. M.; Niklasson, A. M. N.; Challacombe, M. Representation Independent Algorithms for Molecular Response Calculations in Time-dependent Self-consistent Field Theories. J. Chem. Phys. 2009, 130, 054111-054127.

(75) Furche, F.; Ahlrichs, R. Adiabatic Time-dependent Density Functional Methods for Excited State Properties. J. Chem. Phys. 2002, 117, 7433-7448.

(76) Tretiak, S.; Chernyak, V. Resonant Nonlinear Polarizabilities in the Time-dependent Density Functional Theory. J. Chem. Phys. 2003, 119, 8809-8823.

(77) Tommasini, M.; Chernyak, V.; Mukamel, S. Electronic Densitymatrix Algorithm for Nonadiabatic Couplings in Molecular Dynamics Simulations. Int. J. Quantum Chem. 2001, 85, 225-238.

(78) Chernyak, V.; Mukamel, S. Density-matrix Representation of Nonadiabatic Couplings in Time-dependent Density Functional (TDDFT) Theories. J. Chem. Phys. 2000, 8, 3572-3579.

(79) Send, R.; Furche, F. First-order Nonadiabatic Couplings from Time-dependent Hybrid Density Functional Response Theory: Consistent Formalism, Implementation, and Performance. J. Chem. Phys. 2010, 132, 044107-044119.

(80) Mukamel, S. Trees to Trap Photons. Nature 1997, 388, 425427.

(81) Mukamel, S.; Tretiak, S.; Wagersreiter, T.; Chernyak, V. Electronic Coherence and Collective Optical Excitations of Conjugated Molecules. Science 1997, 277, 781-787.

(82) Tretiak, S.; Chernyak, V.; Mukamel, S. Recursive Densitymatrix-spectral-moment Algorithm for Molecular Nonlinear Polarizabilities. J. Chem. Phys. 1996, 105, 8914-8928.

(83) Tretiak, S.; Zhang, W. M.; Chernyak, V.; Mukamel, S. Excitonic Couplings and Electronic Coherence in Bridged Naphthalene Dimers. Proc. Natl. Acad. Sci. U.S.A. 1999, 96, 13003-13008.

(84) Dewar, M. J. S.; Zoebisch, E. G.; Healy, E. F.; Stewart, J. J. P. The Development and Use of Quantum-mechanical molecularmodels.76.AM1 - A New General Purpose Quantum-mechanical Molecular-model. J. Am. Chem. Soc. 1985, 107, 3902-3909.
(85) Tretiak, S.; Chernyak, V.; Mukamel, S. Two-dimensional Realspace Analysis of Optical Excitations in Acceptor-substituted Carotenoids. J. Am. Chem. Soc. 1997, 119, 11408-11419.

(86) Tretiak, S.; Chernyak, V.; Mukamel, S. Collective Electronic Oscillators for Nonlinear Optical Response of Conjugated Molecules. Chem. Phys. Lett. 1996, 259, 55-61.

(87) Wu, C.; Malinin, S. V.; Tretiak, S.; Chernyak, V. Y. Exciton Scattering and Localization in Branched Dendrimeric Structures. Nat. Phys. 2006, 2, 631-635.

(88) Bell, R. J.; Dean, P.; H.-B. D, C. Localization of Normal Modes i n Vitreous Silica, Germania and Beryllium Fluoride. J. Phys. C: Solid State Phys. 1970, 3, 2111-2118.

(89) Taraskin, S. N.; Elliott, S. R. Anharmonicity and Localization of Atomic Vibrations in Vitreous Silica. Phys. Rev. B 1999, 59, 85728585.

(90) Soler, M. A.; Roitberg, A. E.; Nelson, T.; Tretiak, S.; FernandezAlberti, S. Analysis of State-specific Vibrations Coupled to the Unidirectional Energy Transfer in Conjugated Dendrimers. J. Phys. Chem. A 2012, 116, 9802-9810.

(91) Szabo, A. Theory of Fluorescence Depolarization in Macromolecules and Membranes. J. Chem. Phys. 1984, 81, 150-167.

(92) Tretiak, S.; Saxena, A.; Martin, R. L.; Bishop, A. R. Conformational Dynamics of Photoexcited Conjugated Molecules. Phys. Rev. Lett. 2002, 89, 97402-97406.

(93) Wang, L.; Prezhdo, O. V. A Simple Solution to the Trivial Crossing Problem in Surface Hopping. J. Phys. Chem. Lett. 2014, 5, 713-719.

(94) Su, W. P.; Schrieffer, J. R.; Heeger, A. J. Solitons in Polyacetylene. Phys. Rev. Lett. 1979, 42, 1689-1701.

(95) Mostovoy, M. V.; Figge, M. T.; Knoester, J. Disorder-Induced Solitons in Conjugated Polymers. Europhys. Lett. 1997, 38, 687-692.

(96) Knoester, J.; Mostovoy, M. Chapter 3: Disorder and Solitons in Trans-Polyacetylene; In Semiconducting Polymers: Chemistry, Physics and Engineering, Wiley-VCH Verlag GmbH, 1999. 